\title{
The Role of $\mathrm{NH}_{2}$-terminal Positive Charges in the Activity of Inward Rectifier $\mathrm{K}_{\mathrm{ATP}}$ Channels
}

\author{
C.A. Cukras, I. Jeliazkova, and C.G. Nichols \\ Department of Cell Biology and Physiology, Washington University School of Medicine, St. Louis, MO 63110
}

\begin{abstract}
A B S TRACT Approximately half of the $\mathrm{NH}_{2}$ terminus of inward rectifier (Kir) channels can be deleted without significant change in channel function, but activity is lost when more than $\sim 30$ conserved residues before the first membrane spanning domain (M1) are removed. Systematic replacement of the positive charges in the $\mathrm{NH}_{2}$ terminus of Kir6.2 with alanine reveals several residues that affect channel function when neutralized. Certain mutations (R4A, R5A, R16A, R27A, R39A, K47A, R50A, R54A, K67A) change open probability, whereas an overlapping set of mutants (R16A, R27A, K39A, K47A, R50A, R54A, K67A) change ATP sensitivity. Further analysis of the latter set differentiates mutations that alter ATP sensitivity as a consequence of altered open state stability (R16A, K39A, K67A) from those that may affect ATP binding directly (K47A, R50A, R54A). The data help to define the structural determinants of Kir channel function, and suggest possible structural motifs within the $\mathrm{NH}_{2}$ terminus, as well as the relationship of the $\mathrm{NH}_{2}$ terminus with the extended cytoplasmic COOH terminus of the channel.
\end{abstract}

KEY WORDS： $\mathrm{K}^{+}$current $\bullet \mathrm{K}_{\mathrm{ATP}} \bullet \mathrm{PIP}_{2} \bullet \operatorname{Kir} 6.2 \bullet \mathrm{ATP}$

I N T R O D U C T I O N

$\mathrm{K}_{\mathrm{ATP}}$ channels are normally formed as an octameric complex of four Kir6.2 subunits that comprise the pore of the channel, and four regulatory sulfonylurea receptor (SUR)* subunits (Inagaki et al., 1995a, 1997; Clement et al., 1997; Shyng and Nichols, 1997). This complex confers ATP, sulfonylurea, potassium channel opener, and $\mathrm{PIP}_{2}$ sensitivity to a weak inwardly rectifying $\mathrm{K}^{+}$channel (Inagaki et al., 1995a; Fan and Makielski, 1997; Shyng et al., 1997a,b; Baukrowitz et al., 1998; Shyng and Nichols, 1998). Several investigations show that $\mathrm{K}_{\mathrm{ATP}}$ channels formed from Kir6.2 expressed without SUR maintain many of the key properties of the hetero-octameric complex: they are still ATP sensitive (Tucker et al., 1997) and they still respond to PIP $_{2}$ (Baukrowitz et al., 1998; Enkvetchakul et al., 2000), indicating that these properties are inherent to the Kir6.2 subunit.

In the search for where these various modulators are acting, attention has focused on the cytoplasmic domains of Kir6.2. Both $\mathrm{NH}_{2}$ and $\mathrm{COOH}$ termini are cytoplasmic, with the $\mathrm{NH}_{2}$ terminus consisting of $\sim 70$ amino acids, and the large cytoplasmic $\mathrm{COOH}$ terminus consisting of $\sim 220-240$ residues. Although the ATP binding site on Kir6.2 remains elusive, several resi-

Address all correspondence and reprint requests to Colin G. Nichols, Department of Cell Biology and Physiology, Washington University School of Medicine, 660 South Euclid Ave., St. Louis, MO 63110. Fax: (314) 362-7463; E-mail: cnichols@cellbio.wustl.edu

*Abbreviations used in this paper: KIRLI, Kir lipid-interacting; PH, pleckstrin homology; SUR, sulfonylurea receptor. dues in the $\mathrm{COOH}$ terminus, including K185 (Tucker et al., 1997; Reimann et al., 1999a), I182 (Li et al., 2000), R201 (Shyng et al., 2000), and G334 (Drain et al., 1998), have been proposed to be directly involved in ATP binding. Various studies have also indicated several residues in the $\mathrm{COOH}$ termini of Kir channels that may contribute to $\mathrm{PIP}_{2}$ binding (Fan and Makielski, 1997, 1999; Baukrowitz et al., 1998; Shyng and Nichols, 1998; Huang et al., 1998; Liou et al., 1999; Rohacs et al., 1999; Soom et al., 2001). Systematic alanine replacement of all positive residues in the $\mathrm{COOH}$ terminus of Kir6.2 identified residues clustering in two main regions (residues 176-222 and 301-314) that caused a dramatic shift in PIP $_{2}$ activation when mutated (Shyng et al., 2000), and analogous regions of isolated COOHterminal fragments of Kir2.1 are implicated in $\mathrm{PIP}_{2}$ binding (Soom et al., 2001). The role of the $\mathrm{NH}_{2}$ terminus remains incompletely defined. Although $\sim 30$ residues can be cleaved from the $\mathrm{NH}_{2}$ terminus of Kir6.2 without loss of activity, further truncation abolishes channel activity (Koster et al., 1999b). Tolbutamide sensitivity, conferred by coassembly with SUR1, is altered in these truncated constructs, indicating a role for the $\mathrm{NH}_{2}$ terminus in the coupling of the channel to SUR1 (Koster et al., 1999a; Reimann et al., 1999b). $\mathrm{NH}_{2}$-terminal truncations demonstrate a decreased ATP sensitivity (Babenko et al., 1999; Koster et al., 1999b; Reimann et al., 1999b) and residues I49 and $\mathrm{R} 50$ in the $\mathrm{NH}_{2}$ terminus have also been suggested as potential inhibitory ATP binding sites (Tucker et al., 1998; Proks et al., 1999; Reimann et al., 1999b; Tanabe et al., 1999). 
Both the $\mathrm{NH}_{2}$ and $\mathrm{COOH}$ termini are involved in conferring sensitivity of other Kir channels to various agonists, including G-protein sensitivity of Kir3 channels and $\mathrm{pH}$ sensitivity of Kir1.1 channels (Woodward et al., 1997; Schulte et al., 1998). Cyclic-nucleotide gating of CNG channels analogously requires an interaction of the $\mathrm{NH}_{2}$ and $\mathrm{COOH}$ termini (Zagotta and Siegelbaum, 1996; Varnum and Zagotta, 1997). The nonadditive effects of combining mutations in the $\mathrm{NH}_{2}$ and $\mathrm{COOH}$ termini of Kir6.2 on ATP sensitivity has been taken to indicate cooperativity between the two termini (Proks et al., 1999). Tucker and Ashcroft (1999) further demonstrated interaction and binding of the Kir6.2 $\mathrm{NH}_{2}$ terminus to the $\mathrm{COOH}$ termini of Kir6.2, Kir6.1, and Kir2.1 using exogenously produced fusion proteins. They mapped the $\mathrm{NH}_{2}$-terminal interaction region to residues $30-46$, i.e., the most proximal part of the conserved segment (Koster et al., 1999b; Reimann et al., 1999b; Tucker and Ashcroft, 1999). Jones et al. (2001) have recently mapped the complimentary interacting regions in the $\mathrm{COOH}$ terminus and defined three critical segments (170-204, 214-222, and 279-323) that include the regions identified by Shyng et al. (2000) as being involved in PIP $_{2}$ interaction. The identification of interacting portions of Kir6.2 $\mathrm{NH}_{2}$ and $\mathrm{COOH}$ termini leads to the possibility that residues in both the $\mathrm{NH}_{2}$ and $\mathrm{COOH}$ termini are involved in determining the sensitivity of the channel to agonists such as ATP and PIP $_{2}$. We have recently defined a probable $\alpha$-helix in Kir channel $\mathrm{COOH}$-terminal domains that is similar to the $\mathrm{COOH}-$ terminal $\alpha$-helix of pleckstrin homology $(\mathrm{PH})$ domains. We hypothesize that Kir channels contain a conserved lipid interaction (KIRLI) domain, that may be related to $\mathrm{PH}$ domains, with structural elements defined by Tucker and Ashcroft (1999) and Jones et al. (2001) contributed by both $\mathrm{NH}_{2^{-}}$and $\mathrm{COOH}$-terminal regions (Cukras et al., 2002).

To generate a primary dataset for consideration of $\mathrm{NH}_{2}$-terminal residues in channel function, we have now systematically mutated each positive charge to alanine. We report several residues in the $\mathrm{NH}_{2}$ terminus that affect the apparent ATP sensitivity or the open probability when mutated. Furthermore, we distinguish between residues that might be involved in ATP binding and those that affect the open state stability of the channel, and are thus likely to be involved in $\mathrm{PIP}_{2}$ interaction.

M A T E R I L S A N D M E T H O D S

\section{Molecular Biology}

Point mutations were prepared by overlap extension at the junctions of the relevant residues by sequential PCR. Resulting PCR products were subcloned into pCMV6b vector. Before transfection, constructs were sequenced to verify the correct mutations.

\section{Expression in COSm6 Cells}

COSm6 cells were plated at a density of $\sim 2.5 \times 10^{5}$ cells per well (30 mm six-well dishes) and cultured in Dulbecco's Modified Eagle Medium plus $10 \mathrm{mM}$ glucose (DMEM-HG), supplemented with FCS $(10 \%)$. The next day, cells were transfected by adding FUGENE and $1 \mu \mathrm{g}$ each of pCMV6b-Kir6.2 or mutant isoforms, pECE-SUR1 cDNA, and pECE-GFP (green fluorescent protein) directly to the media. The cells were replated the next day onto coverslips for patch-clamping. All experiments were performed 24-48 h posttransfection.

\section{Patch-clamp Measurements}

Patch-clamp experiments were made at room temperature, in an oil-gate chamber that allowed rapid exchange of bathing solution by moving patches from one channel to another (Lederer and Nichols, 1989). Micropipettes were pulled from thin-walled glass (WPI, Inc.) on a horizontal puller (Sutter Instrument Co.). Electrode resistance was typically $0.5-1 \mathrm{M} \Omega$ when filled with K-INT solution (below). Inside-out patches were voltage-clamped at -50 $\mathrm{mV}$ with an Axopatch 1B amplifier (Axon Instruments, Inc.). Standard bath and pipette solutions (K-INT) had the following composition: $140 \mathrm{mM} \mathrm{KCl}, 10 \mathrm{mM}$ K-HEPES, 1 mM K-EGTA, pH 7.3. PIP $_{2}$ was bath sonicated in ice for $30 \mathrm{~min}$ before use. ATP was added as the potassium salt. All currents were measured at a membrane potential of $-50 \mathrm{mV}$, and inward currents at this voltage are shown as upward deflections. Data were filtered at $0.5-3 \mathrm{kHz}$, digitized at 22 $\mathrm{kHz}$ (Neurocorder; Neurodata) and stored on videotape. Experi-
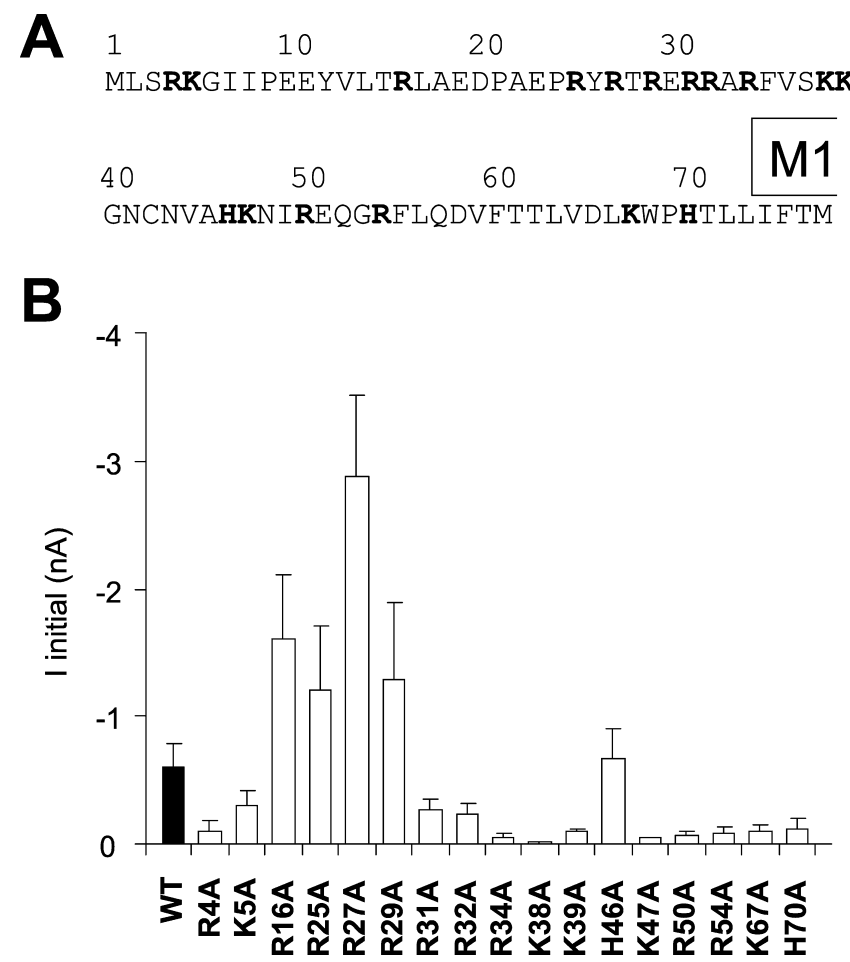

Figure 1. Alanine scanning mutagenesis of $\mathrm{NH}_{2}$-terminal basic residues of Kir6.2. (A) Each positive charged residue in the $\mathrm{NH}_{2}$ terminus (bold), up to the beginning of the M1 transmembrane domain, was individually mutated to alanine. (B) Initial current $\left(\mathrm{I}_{\text {initial }}\right)$ in macropatches isolated from COSm6 cells cotransfected with mutant Kir6.2 subunits (as indicated) + SUR1 (mean \pm SEM, $n \geq 3$ in each case). See MATERIALS AND METHODS for details. 

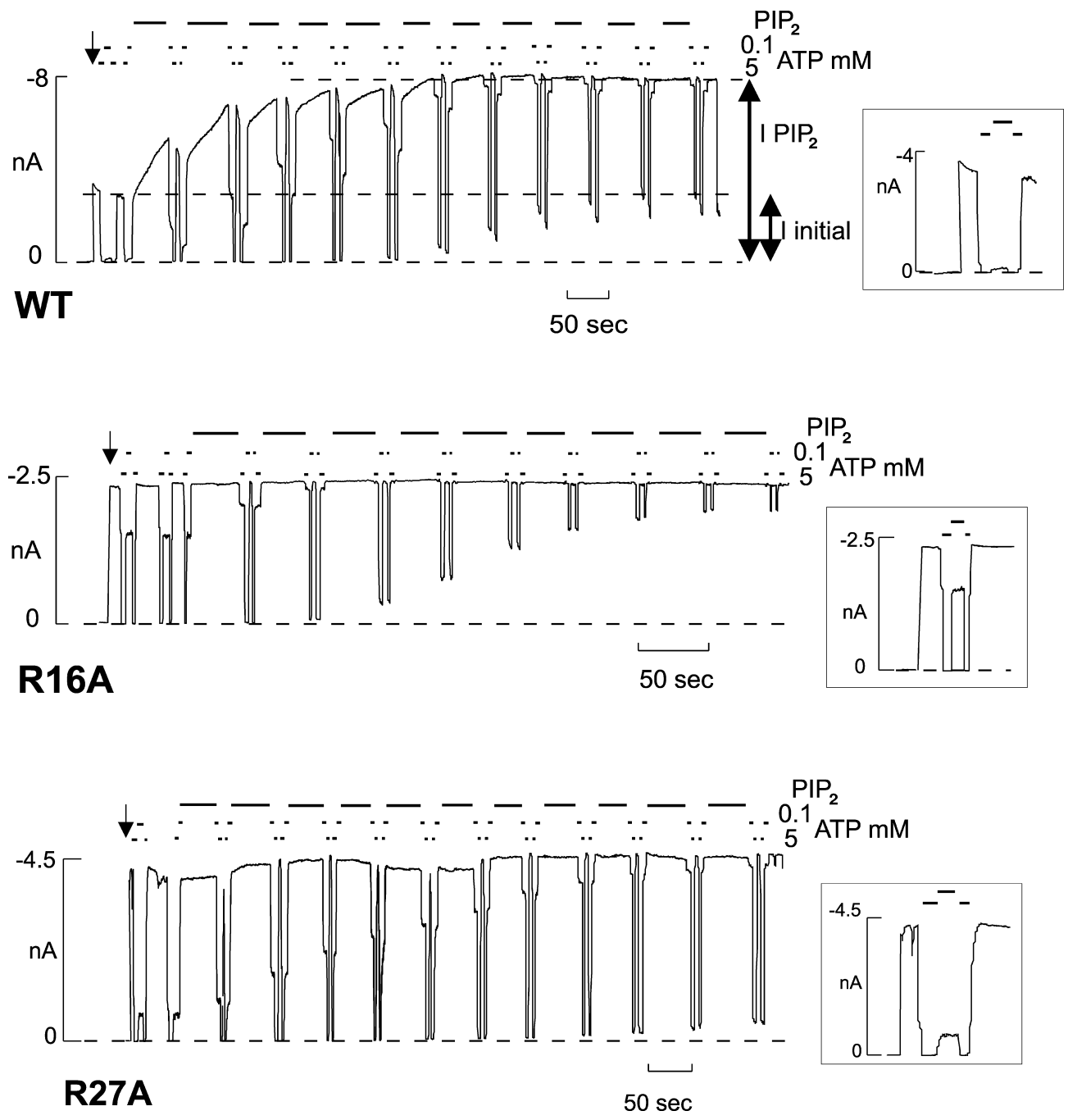

FIgURE 2. Representative currents recorded from inside-out membrane patches containing Kir6.2 WT, Kir6.2[R16A], Kir6.2[R27A], coexpressed with SUR1. In this and subsequent figures, the patch was excised at the arrow, and the bars indicate the application of $\mathrm{PIP}_{2}(5 \mu \mathrm{g} / \mathrm{ml}$, unless indicated) or different [ATP] (as shown). Solutions were switched by moving the patch from one lane of the oil-gate chamber to another. The lower dashed line indicates zero current. $\mathrm{I}_{\text {initial }}$ and $\mathrm{I}_{\mathrm{PIP}_{2}}$, used to determine $\mathrm{P}_{\text {o,zero }}$ (see MATERIALS AND METHODS), are indicated by additional dashed lines. The inserts in this and Fig. 3 show expanded records from the first 50-100 s after patch excision, from which ATP sensitivity and open probability (NA method) were estimated. ments were replayed onto a chart recorder, or digitized into a computer using Axotape software (Axon Instruments, Inc.) and analyzed off-line using Microsoft Excel. Wherever possible, data are presented as mean \pm SEM. Microsoft Solver was used to fit data by a least-square algorithm.

\section{Interpretation of $\mathrm{PIP}_{2}$ Response Data}

Wild-type (WT) $\mathrm{K}_{\mathrm{ATP}}$ (Kir6.2 + SUR1) channels have an intrinsic open probability in the absence of ATP $\left(\mathrm{P}_{\mathrm{o}, \text { zero }}\right)$ of $\sim 0.4$ and are half maximally inhibited at an ATP concentration $\left(\mathrm{K}_{1 / 2, \mathrm{ATP}}\right)$ of $\sim 10 \mu \mathrm{M}$ (Enkvetchakul et al., 2000; Inagaki et al., 1995b). Many mutations of Kir6.2 residues, or exposure of channels to cytoplasmic $\mathrm{PIP}_{2}$, cause changes in both $\mathrm{P}_{\mathrm{o}, \text { zero }}$ and ATP sensitivity. In many cases, $\mathrm{P}_{\mathrm{o} \text {,zero }}$ and $\mathrm{K}_{1 / 2, \mathrm{ATP}}$ are strongly correlated, and this correlation can be explained by assuming that the action of ATP is on the closed channel, such that both $\mathrm{P}_{\mathrm{o} \text {,zero }}$ and $\mathrm{K}_{1 / 2, \mathrm{ATP}}$ are increased when open state stability is increased, for example, by addition of $\mathrm{PIP}_{2}$ (Baukrowitz et al., 1998; Shyng and Nichols, 1998; Enkvetchakul et al., 2000). Mutations could affect the response of the channel to $\mathrm{PIP}_{2}$ by altering either the affinity or availability of a $\mathrm{PIP}_{2}$ binding site, or by altering the coupling of $\mathrm{PIP}_{2}$ binding to open state stability. These possibilities are experimentally unresolvable and therefore, in the present experiments, we interpret reduced intrinsic activity and greater response to $\mathrm{PIP}_{2}$ as indicating that mutations either affect $\mathrm{PIP}_{2}$ binding directly, or the translation of this binding into an effect on channel open state stability.

\section{Data Analysis}

Off-line analysis was performed using ClampFit and Microsoft Excel programs. Two approaches were used to estimate $\mathrm{P}_{\mathrm{o}, \mathrm{zero}}$, the initial open probability (in zero ATP), after excision of isolated membrane patches containing multiple channels. (1, $\mathrm{PIP}_{2}$ method) $\mathrm{PIP}_{2}$ was added to the patch until the current reached a saturating level $\left(\mathrm{I}_{\mathrm{PIP}_{2}}\right)$. This was assumed to represent a maximum $\mathrm{P}_{\text {o,zero }}$ of $\sim 0.9$ (Enkvetchakul et al., 2000). The fold increase in current was calculated (fold increase $=I_{\text {initial }} / I_{\text {PIP2 }}$, see Fig. 2) and the $P_{o, z e r o}$ was estimated from the following equation: $\mathrm{P}_{\mathrm{o} \text {,zero }}=0.9 /$ (fold increase). (2, NA method) Additionally, mean $\mathrm{P}_{\mathrm{o} \text {,zero }}$ was estimated from stationary fluctuation analysis of macroscopic currents (Neher and Stevens, 1977; Sigworth, 1980) on short $(<1 \mathrm{~s})$ recordings of currents in zero [ATP] and in $5 \mathrm{mM}$ [ATP]. Currents $(4 \mathrm{pA}<$ mean current $<4 \mathrm{nA}$, at $-50 \mathrm{mV}$, corresponding to $\sim 1-1,000$ channels) were filtered at $1 \mathrm{kHz}$ and digitized at $3 \mathrm{kHz}$ with 12-bit resolution. Mean patch current (I), and variance $\left(\sigma^{2}\right)$ in the absence of ATP were obtained by subtraction of the mean current and variance in $5 \mathrm{mM}$ ATP (i.e., assuming all channels closed), respectively. Single channel current (i) was assumed to be $-3.75 \mathrm{pA}$, corresponding to WT single channel conductance of $75 \mathrm{pS}$. $\mathrm{P}_{\mathrm{o}, \text { zero }}$ was then estimated from the following equation: $P_{\text {o,zero }}=1-(\sigma 2 /[i])$. Although the $\mathrm{P}_{\mathrm{o}, \text { zero }}$ estimated from noise analysis (NA) was systematically higher than that from $\mathrm{PIP}_{2}$ response, it is apparent in Fig. 4 that both methods gave correlated estimates of $\mathrm{P}_{\mathrm{o}, \mathrm{zero}}$. Therefore, al- 

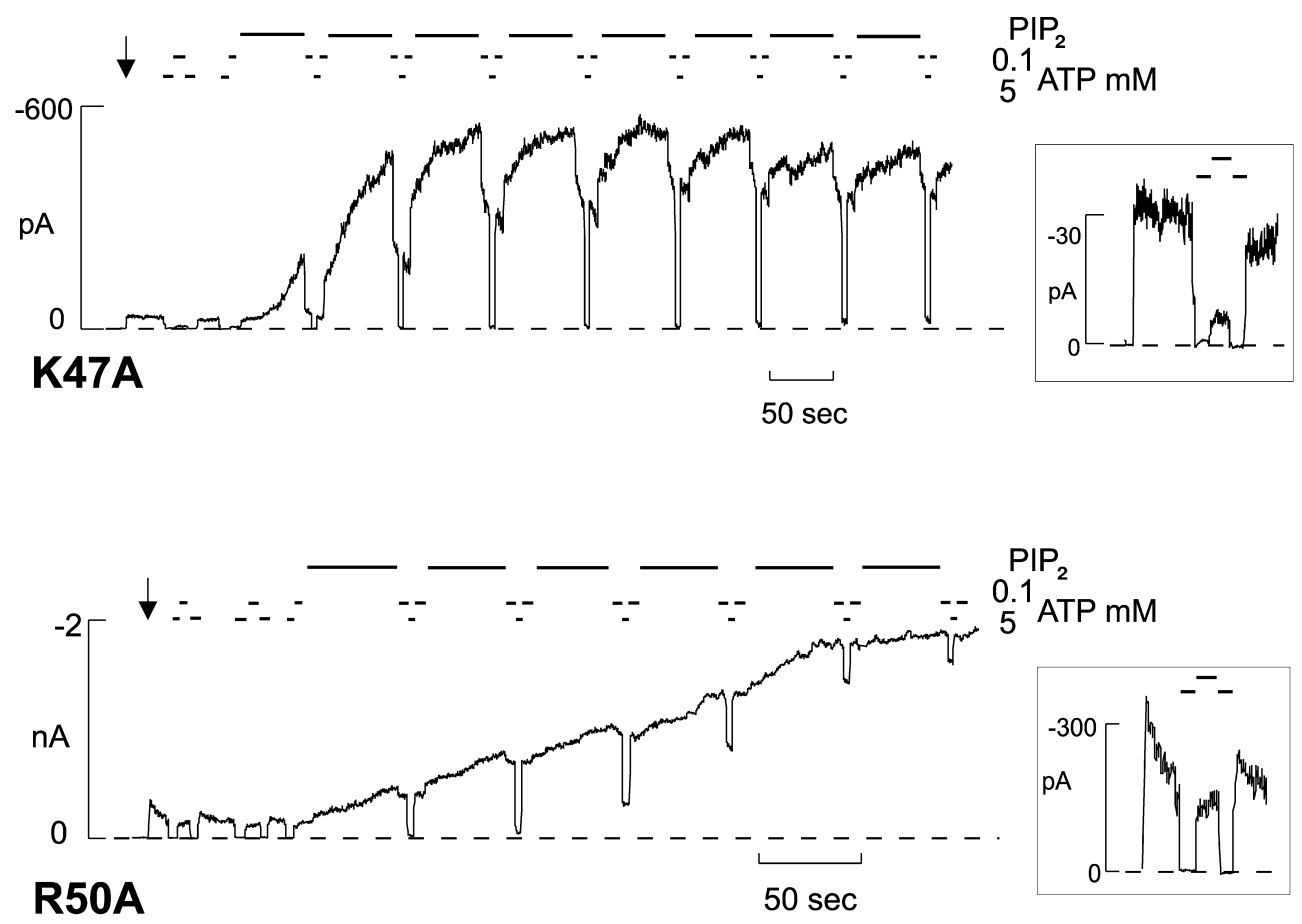

FiguRE 3. Representative currents recorded from inside-out membrane patch containing Kir6.2[K47A], Kir6.2[R50A],

or

Kir6.2[R54A] coexpressed with SUR1. The dashed line indicates zero current.

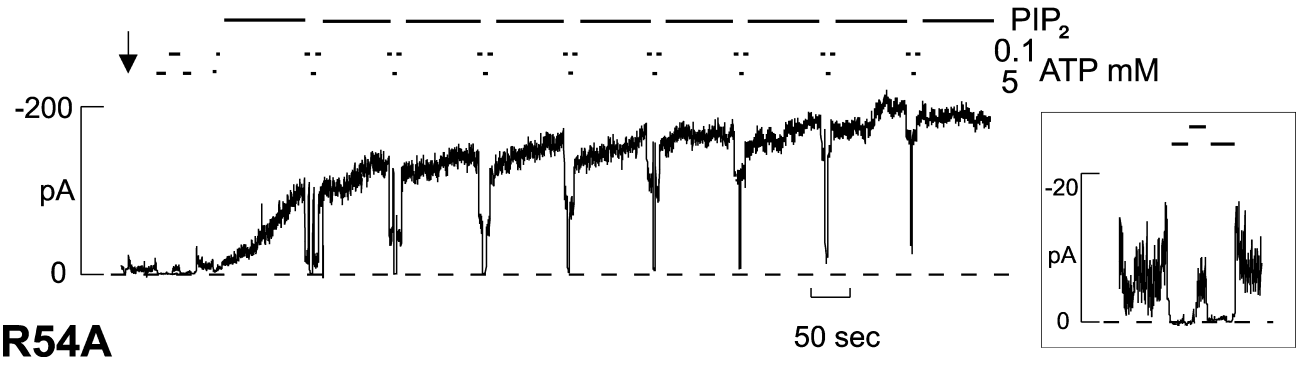

though absolute $\mathrm{P}_{\mathrm{o}, \text { zero }}$ estimates are unlikely to be accurate, we have reasonable confidence in comparing relative $\mathrm{P}_{\text {o,zero }}$ between mutants. Since the $\mathrm{PIP}_{2}$ response (lower estimate) will actually report an upper limit for $\mathrm{P}_{\mathrm{o} \text {,zero }}$, we utilize these values for examination of $\mathrm{P}_{\text {o,zero }}-\mathrm{K}_{1 / 2 \mathrm{ATP}}$ relationships (Fig. 2).

ATP sensitivity was estimated from least squares fits of the Hill equation to the currents in $0,0.1$, and $5 \mathrm{mM}$ ATP immediately after patch excision (see insets to Figs. 2 and 3): $\mathrm{I}_{\text {rel }}=1 /(1+$ $\left.\left\{[\mathrm{ATP}] / \mathrm{K}_{1 / 2}\right\}^{\mathrm{H}}\right)$, where $\mathrm{I}_{\text {rel }}$ is the mean current in a given ATP concentration ([ATP $]$ ) divided by the mean current in zero ATP, $\mathrm{K}_{1 / 2}$ is the [ATP] causing half-maximal inhibition, and $\mathrm{H}$ is the Hill coefficient (fixed at 1.3, Shyng et al., 2000).

\section{R E S U L T S}

\section{Alanine Scan of $\mathrm{NH}_{2}$ Terminus Identifies Residues That Affect Open Probability $\left(P_{o, z e r o}\right)$}

There are 17 positive charges ( $\mathrm{R}$, arginine; $\mathrm{K}$, lysine; $\mathrm{H}$, histidine) in the $\mathrm{NH}_{2}$ terminus of Kir6.2 (Fig. $1 \mathrm{~A}$ ) any of which could contribute to interaction with either $\mathrm{PIP}_{2}, \mathrm{ATP}$, or both. To assess the role of these charged residues in $\mathrm{K}_{\mathrm{ATP}}$ channel function, we mutated each one to alanine and examined channel properties in in- side-out membrane patches. Fig. 1 B shows a summary of current density in patches from COSm6 cells expressing each mutant channel. In contrast to the severe detrimental effects of charge neutralization in some residues in the $\mathrm{COOH}$ terminus (Shyng et al., 2000), all of the $\mathrm{NH}_{2}$-terminal mutants generated measurable $\mathrm{K}^{+}$currents. Wide variability of $\mathrm{I}_{\text {initial }}$ is reflective of variability of $\mathrm{P}_{\mathrm{o} \text {,zero }}$ (see below, Fig. 4, inset).

Figs. 2 and 3 show representative recordings for various mutations. From such recordings we estimated initial $\mathrm{P}_{\mathrm{o} \text { zero }}$ and ATP sensitivity after patch excision. Initial $\mathrm{P}_{\text {o,zero }}$ was estimated in two ways: (a) by measuring the response to $\mathrm{PIP}_{2}$ and (b) by noise analysis (see MATERIALS AND METHODS). WT channels typically have a $\mathrm{P}_{\mathrm{o} \text { zero }}$ around 0.4 (Fig. 2, top; Shyng and Nichols, 1998; Enkvetchakul et al., 2000; Shyng et al., 2000). After application of $\mathrm{PIP}_{2}$, WT open probability increases and saturates at $\sim 0.9$ (Shyng and Nichols, 1998; Enkvetchakul et al., 2000; Shyng et al., 2000), such that macroscopic currents approximately double. Fig. 4 summarizes the estimates of $\mathrm{P}_{\mathrm{o} \text {,zero }}$ using the 


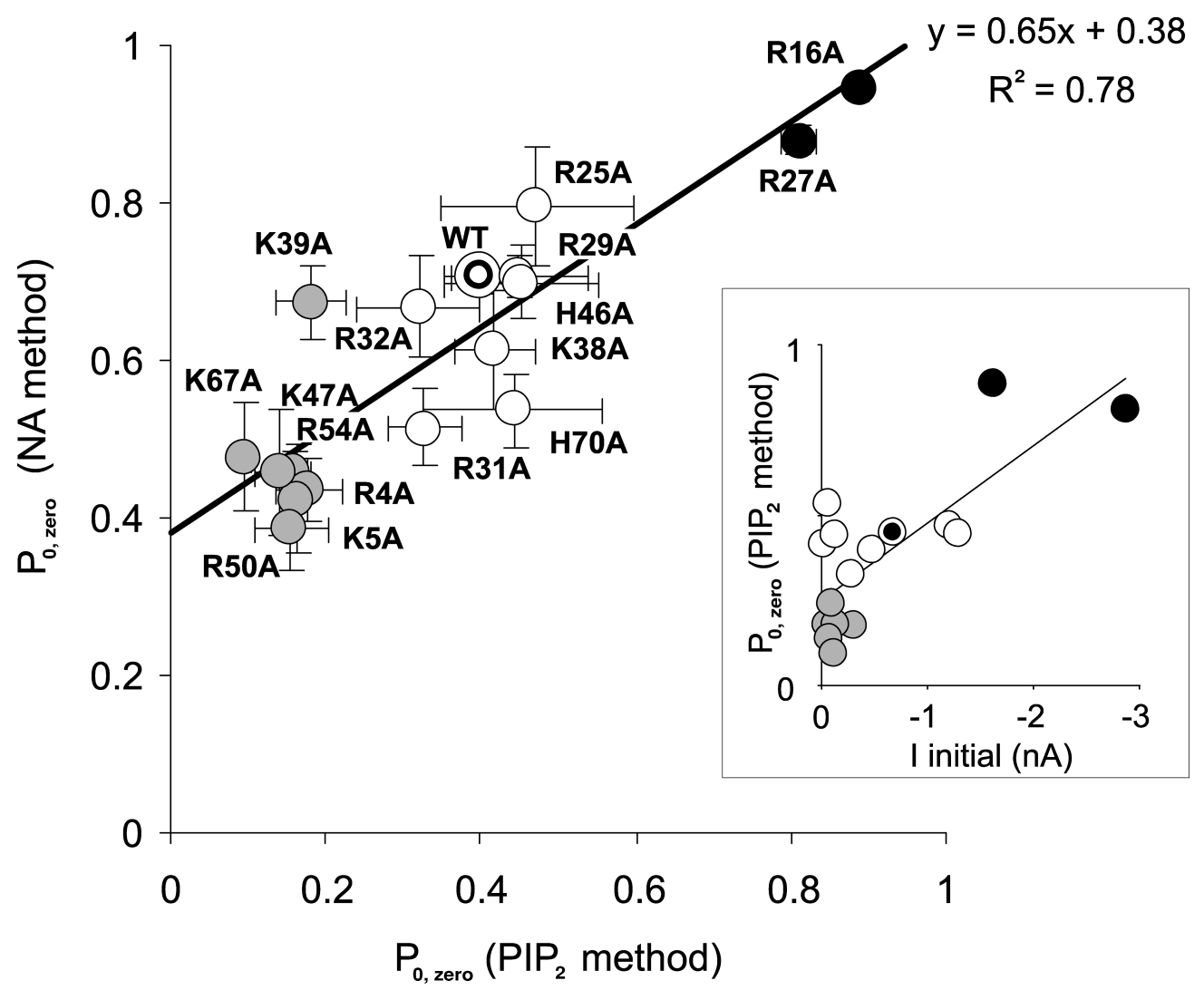

Figure 4. Initial open probability in zero ATP $\left(\mathrm{P}_{\mathrm{o}, \text { zero }}\right)$ obtained from noise analysis (NA method, mean \pm SEM, $n=$ 3-8) plotted versus $\mathrm{P}_{\mathrm{o} \text {,zero }}$ obtained from subsequent increase in current following $\mathrm{PIP}_{2}$ addition $\left(\mathrm{PIP}_{2}\right.$ method, mean $\pm \mathrm{SEM})$. The solid line is a least squares fit to the mean data as indicated. The insert shows mean $\mathrm{P}_{\mathrm{o} \text {,zero }}$ $\left(\mathrm{PIP}_{2}\right.$ method) plotted against $\mathrm{I}_{\text {initial }}($ from Fig. $1 \mathrm{~B}$ ) for each mutant. two independent methods. Each method clearly gives correlated estimates, even though $\mathrm{P}_{\text {o,zero }}$ as estimated by noise analysis is systematically higher than estimated by the $\mathrm{PIP}_{2}$ method, probably due to inevitable underestimation of noise due to filtering. Although the $\mathrm{P}_{\mathrm{o} \text {,zero }}$ of many mutations were not significantly different from WT, some (R16A, R27A, Fig. 2) have a much higher $\mathrm{P}_{\mathrm{o} \text {,zero }}$, whereas others (R4A, R5A, R39A, K47A, R50A, R54A, K67A) have a significantly lower $\mathrm{P}_{\mathrm{o}, \mathrm{zero}}$. The inset of Fig. 4 demonstrates that the initial current following patch excision is also reasonably well correlated with $\mathrm{P}_{\text {ozero }}$.

The behavior of mutant R34A was somewhat unique in that the channels showed high $\mathrm{P}_{\mathrm{o}, \mathrm{zero}}(0.86 \pm 0.08$, $n=4)$, as estimated by noise analysis, but ran down very quickly (Fig. $5 \mathrm{~A}$ ). After $\mathrm{PIP}_{2}$ addition there was no increase in channel current (Fig. 5 B), precluding estimate of $\mathrm{P}_{\mathrm{o} \text { zero }}$ (see Discussion).

\section{Alanine Scan of $\mathrm{NH}_{2}$ Terminus Identifies Residues That Affect ATP Sensitivity}

We evaluated the ATP sensitivity for each mutant (Fig. 6 A), after patch excision, from records such as those in Figs. 2 and 3. In addition to the previously identified R50A (Proks et al., 1999), R16A is considerably ( 10fold) less sensitive to ATP than WT channels, and R27A, $\mathrm{K} 47 \mathrm{~A}$, and R54A mutants are also, though less markedly, reduced in ATP sensitivity $(\mathrm{P}<0.05)$ (Fig. 2).
A mutation can change ATP sensitivity by directly reducing the affinity of binding, by affecting the binding site allosterically, or by affecting the open state stability of the channel. We have previously proposed a model that quantitatively describes $\mathrm{K}_{\mathrm{ATP}}$ channel behavior over a wide range of conditions and mutations (Model V, see inset Fig. 6) (Enkvetchakul et al., 2000), except for mutations that have extremely high open state stability (e.g., Kir6.2[L164C]; Enkvetchakul et al., 2001). An essential feature of such a model is that ATP predominantly acts to stabilize a closed state and, in consequence, the apparent ATP sensitivity can be reduced either by shifting the equilibrium between $\mathrm{C}$ and $\mathrm{O}$ states toward the open state or by reduction of the ATP binding. Fig. $6 \mathrm{~B}$ plots the measured $\mathrm{K}_{1 / 2, \mathrm{ATP}}$ as a function of the estimated $\mathrm{P}_{\text {o,zero }}$ (from $\mathrm{PIP}_{2}$ response) for each mutant. The bold line indicates the prediction of Model $\mathrm{V}$ for varying intrinsic open state stability (i.e., the $\mathrm{K}_{\mathrm{CO}}$ equilibrium constant). From this analysis it is apparent that many mutations actually cluster very close to WT and most of the remainder are distributed along the predicted curve (mutations indicated in gray are shifted significantly $(\mathrm{P}<0.05)$ along the $\mathrm{P}_{\mathrm{o}, \mathrm{zero}}$ axis from $\left.\mathrm{WT}\right)$. This implies that these mutations act to alter the intrinsic open state stability, and in doing so, ATP sensitivity changes according to the relationship indicated. We further estimated whether the measured mean $\mathrm{K}_{1 / 2, \mathrm{ATP}}$ of each mutant was different from that predicted given the mean $\mathrm{P}_{\mathrm{o}, \mathrm{zero}}$. This 

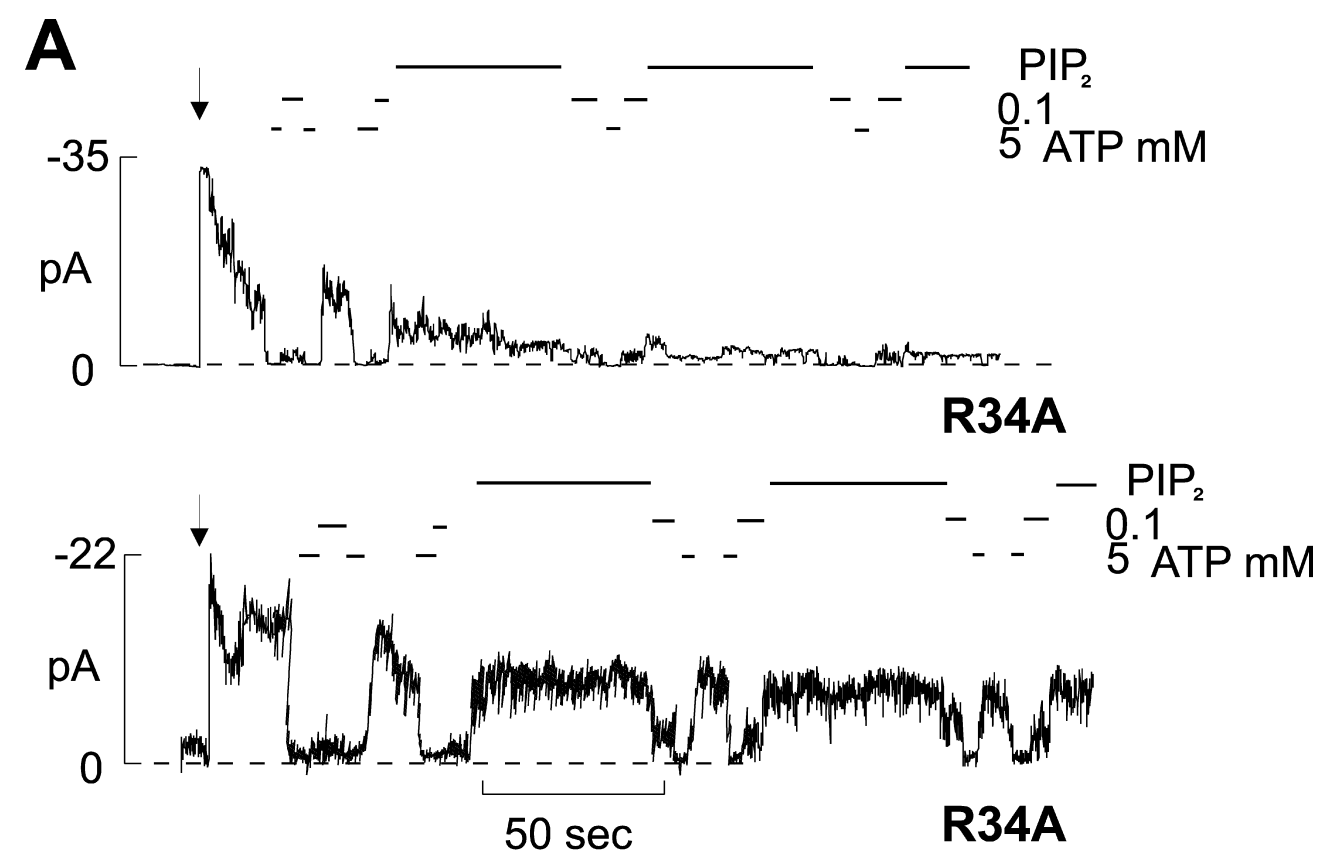

Figure 5. (A) Representative currents recorded from inside-out membrane patches containing Kir6.2[R34A] coexpressed with SUR1. The dashed line indicates zero current. (B) Mean current (in zero ATP) versus time after application of $\mathrm{PIP}_{2}$ for Kir6.2 WT coexpressed with SUR1 and for Kir6.2[R34A] coexpressed with SUR1.

B

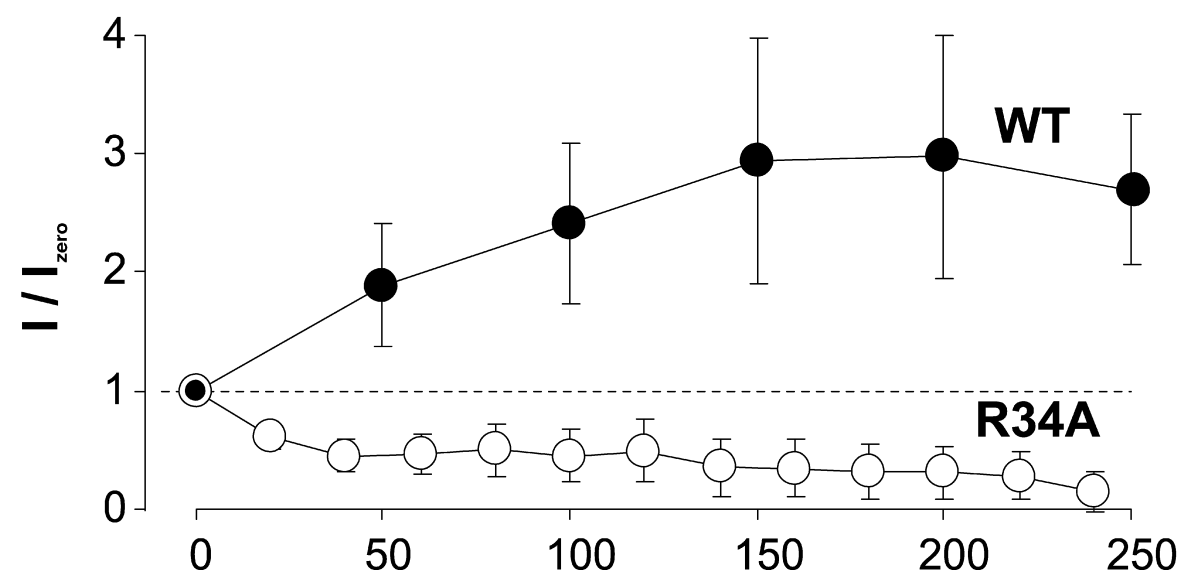

further identified three residues (K47A, R50A, and R54A, black in Fig. 6 B) that are significantly $(\mathrm{P}<0.05)$ less sensitive to ATP than predicted from $\mathrm{P}_{\mathrm{o}, \mathrm{zero}}$. A reduction of ATP sensitivity without increase in $\mathrm{P}_{\mathrm{o} \text {,zero }}$ implicates these residues as potentially contributing to ATP binding itself (see DISCUSSION).

D I S C U S S I O N

\section{Distinct Phenotypic Consequences of Charge Neutralization}

$\mathrm{PIP}_{2}$ and other negatively charged phospholipids increase the open state stability of Kir channels (Hilgemann and Ball, 1996; Fan and Makielski, 1997). The molecular mechanism by which this occurs is not resolved, but it is likely that electrostatic interactions between a Kir cytoplasmic domain and phospholipids in the membrane stabilizes the open state of the channel
(Fan and Makielski, 1997; Huang et al., 1998; Shyng et al., 2000; Soom et al., 2001; Cukras et al., 2002). ATP and $\mathrm{PIP}_{2}$ are negative heterotropic regulators of $\mathrm{K}_{\mathrm{ATP}}$ channels, such that binding of ATP or $\mathrm{PIP}_{2}$ stabilizes the closed channel or the open channel respectively (Shyng and Nichols, 1998; Enkvetchakul et al., 2000). Therefore, treatment of membrane patches with $\mathrm{PIP}_{2}$ leads to an increase of $\mathrm{P}_{\mathrm{o} \text {,zero }}$ and decrease of ATP-sensitivity (Baukrowitz et al., 1998; Shyng and Nichols, 1998; Enkvetchakul et al., 2000; Shyng et al., 2000).

Systematic neutralization of positively charged residues in the COOH terminus of Kir6.2 subunits demonstrated distinct sets of residues that affect $\mathrm{PIP}_{2}$-sensitivity and ATP sensitivity (Shyng et al., 2000). The present data demonstrates that distinct sets of basic residues in the $\mathrm{NH}_{2}$ terminus also contribute to ATP sensitivity and $\mathrm{PIP}_{2}$ sensitivity. In every case, channel activity was present 


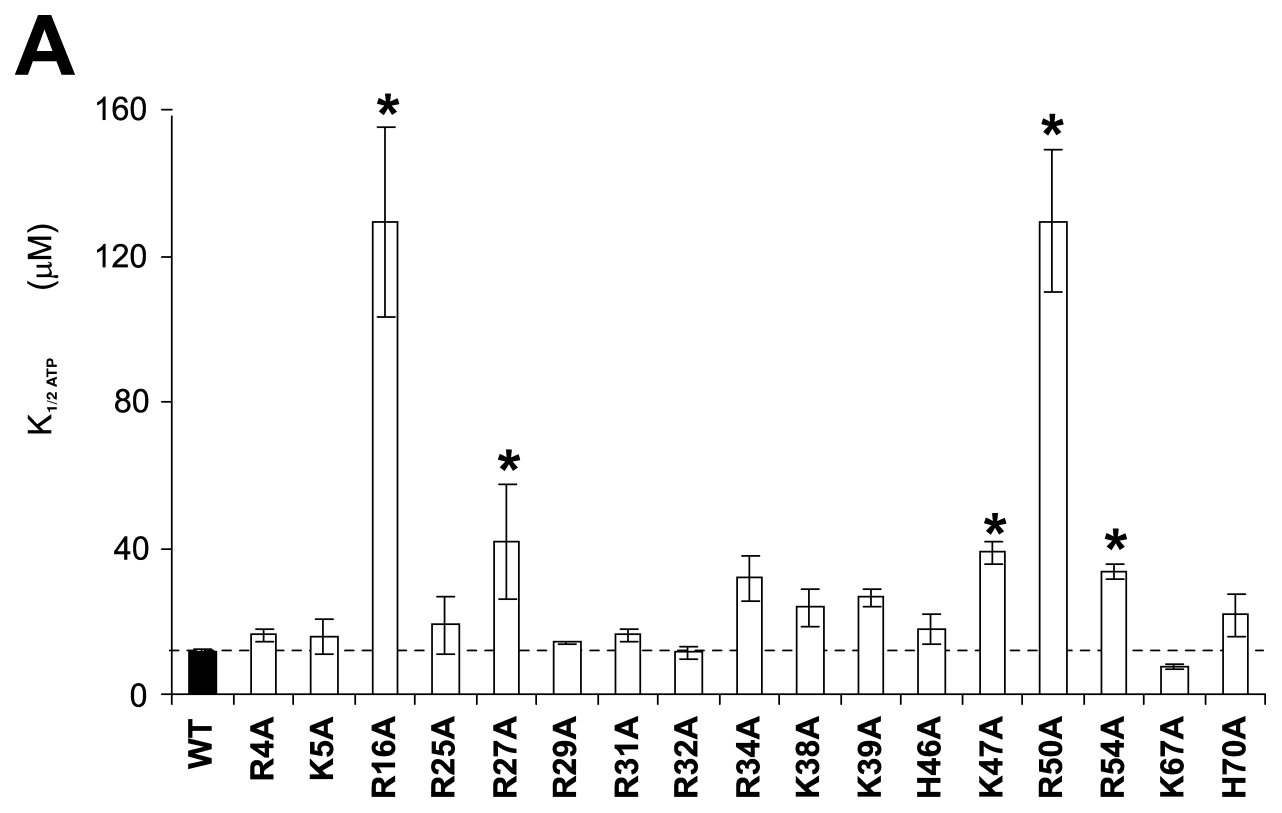

B

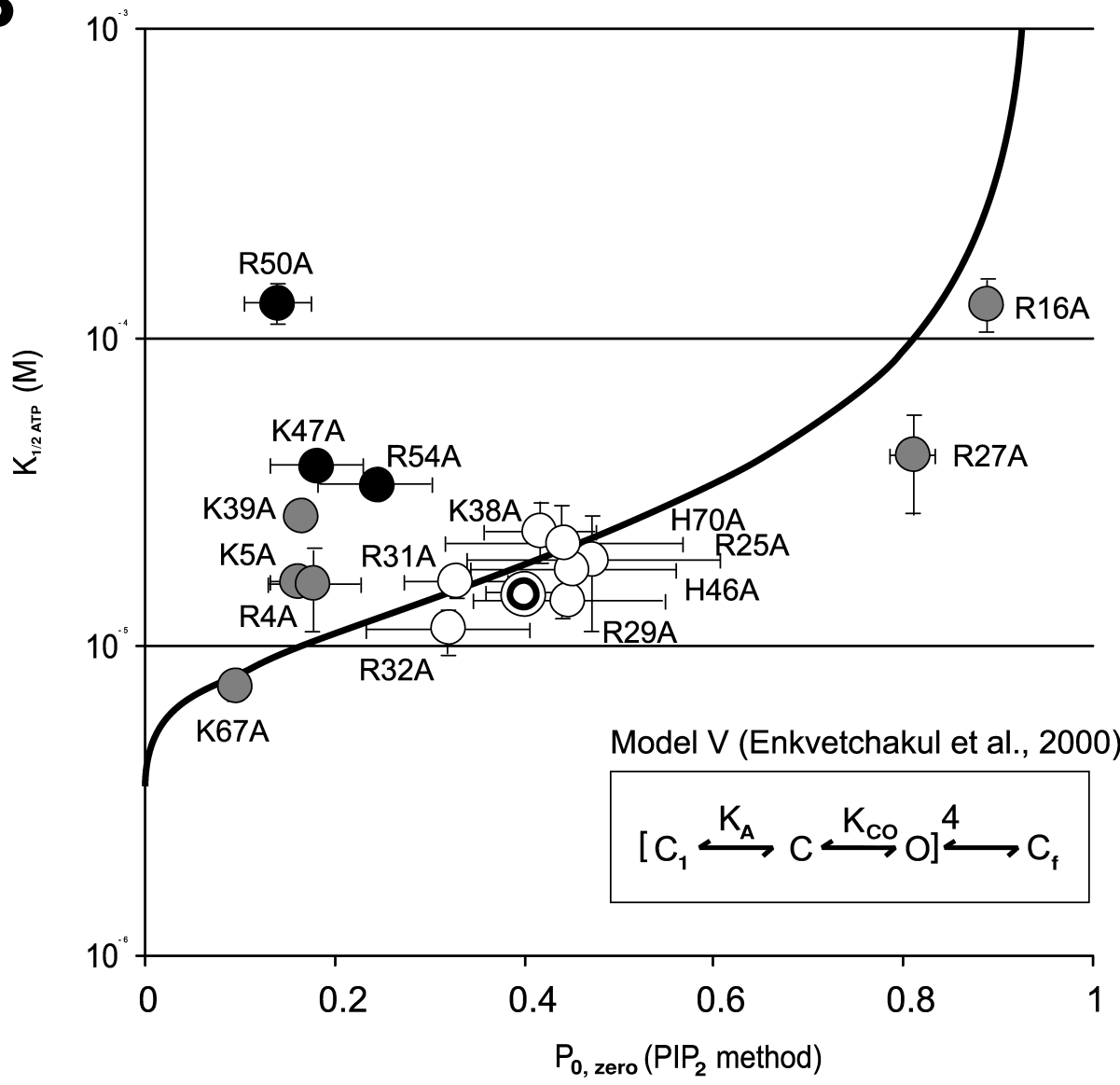

Figure 6. (A) Mean $\mathrm{K}_{1 / 2 \mathrm{ATP}}$ for each mutant immediately following patch excision (mean \pm SEM, $n=3-9$, asterisk indicates less ATP-sensitive than WT, P < $0.05)$. Dose-response curves were obtained from recordings like those in Figs. 2 and 3 and were fit using a Hill equation (relative current $\left.=1 /\left\{1+\left([\mathrm{ATP}] / \mathrm{K}_{1 / 2}\right)^{\mathrm{H}}\right\}\right)$ with $\mathrm{K}_{1 / 2, \mathrm{ATP}}=$ [ATP] causing half-maximal inhibition and $\mathrm{H}=$ 1.3. (B) $\mathrm{K}_{1 / 2, \mathrm{ATP}}$ versus $\mathrm{P}_{\mathrm{o}, \text { zero }}$ for each mutant (mean \pm SEM, $n=$ 3-9; for each parameter). The double circle represents mean values for WT. For different Kir6.2 mutant constructs, gray/ black indicates $\mathrm{P}_{\mathrm{o} \text {,zero }}$ significantly different than WT $(\mathrm{P}<0.05)$. The heavy line represents predicted $\mathrm{P}_{\mathrm{o} \text {,zero }}$ versus $\mathrm{K}_{1 / 2, \text { ATP }}$ relationship calculated from Model V of Enkvetchakul et al. (2000, see insert), black indicates mutants with $\mathrm{K}_{1 / 2, A T P}$ significantly different than that predicted from the estimated $\mathrm{P}_{\text {o,zero }}(\mathrm{P}<$ $0.05)$.

in excised patches (Fig. $1 \mathrm{~B}$ ), and thus none of the mutations caused global structural changes that made subunits nonfunctional. Of 15 positively charged residues, mutation of seven (R4A, K5A, K39A, K47A, R50A, R54A, K67A) significantly reduced $\mathrm{P}_{\text {o,zero }}$ (Fig. 4). Two muta- tions (R16A, R27A) led to increased open probability, and an expected decrease of ATP sensitivity. Thus, these two sets of mutations reflect a decrease, or increase, respectively, in the intrinsic open state stability, which may reflect altered affinity for binding of $\mathrm{PIP}_{2}$ (see below). 


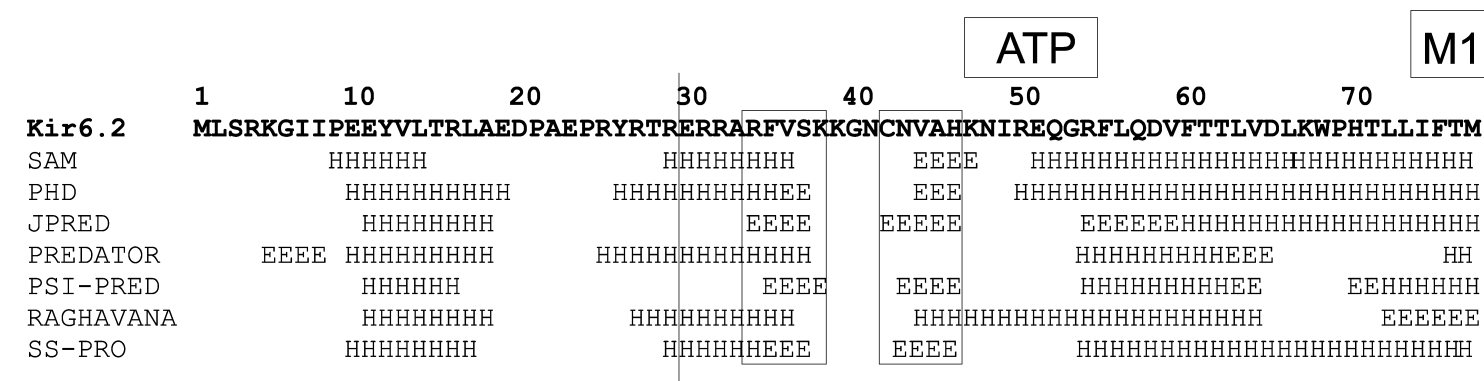

FigURE 7. Computer-predicted secondary structures (E, $\beta$-strand; $\mathrm{H}$, helix) of conserved $\mathrm{NH}_{2}$-terminal (residues 1-77) region of Kir6.2 using multiple alignments with other inward rectifiers, generated by various algorithms indicated on left. The vertical line indicates the approximate boundary between essential (i.e., conserved) and nonessential regions. Putative $\beta$-strands are boxed, and the loop proposed to contribute to ATP binding is indicated above the sequence.

Three mutations (K47A, R50A, R54A), which cluster closely together, caused a decrease in ATP sensitivity that was not correlated with increased $\mathrm{P}_{\mathrm{o}, \mathrm{zero}}$ (Fig. $6 \mathrm{~B}$ ). These mutations thus represent possible ATP binding site mutations. These results support and extend the previous suggestion that R50 is involved in ATP binding (Tucker et al., 1998; Reimann et al., 1999b), either directly by forming part of the binding site, or allosterically (Tucker et al., 1998; Proks et al., 1999) by affecting ATP binding. That the $\mathrm{P}_{\text {o,zero }}$ of each of these mutants is actually significantly lower than that of WT (Fig. 6) is consistent with these residues also being involved in the interaction of the channel with $\mathrm{PIP}_{2}$. In general, our findings from an alanine scan of the $\mathrm{COOH}$ terminus indicate that there are likely to be distinct, yet allosterically coupled and possibly overlapping, binding sites for ATP and PIP $_{2}$ (Shyng et al., 2000). It is possible, therefore, that while the majority of the residues affect only one or the other sites, a few residues might be involved either in the physical overlap of the two sites or be directly involved in one site while allosterically affecting the other. This is the role/location that we previously proposed for R201 (Shyng et al., 2000) and now propose for K47, R50, and R54.

Finally, the R34A mutation demonstrated a novel phenotype. Channel activity was evident after patch excision, but run-down was rapid, and not prevented by $\mathrm{PIP}_{2}$. This effect is distinct from the effect of $\mathrm{COOH}$-terminal mutations (e.g., R176A) that reduce $\mathrm{PIP}_{2}$ sensitivity, putatively by affecting PIP2 binding. In the case of R176A, mutation results in lower intrinsic $\mathrm{P}_{\mathrm{o}, \text { zero }}$, but with a dramatic increase in channel activity on exposure to $\mathrm{PIP}_{2}$. In the case of R34A, the intrinsic $\mathrm{P}_{\mathrm{o} \text {,zero }}$ is not obviously reduced, but there is no increase in current after $\mathrm{PIP}_{2}$ exposure. Conceivably, this mutation induces a rapid rundown by some different, unknown, mechanism.

\section{Effects of Truncation Experiments}

Previous truncation experiments have shown that deletion of the first 20-30 amino acids of various inward rectifiers may be possible, without loss of channel function (Zhou et al., 1994; Boim et al., 1995; Babenko et al., 1999; Koster et al., 1999b; Reimann et al., 1999b). In the case of Kir6.2, significantly higher $\mathrm{K}_{1 / 2 \mathrm{ATP}}$ and $\mathrm{P}_{\mathrm{o} \text {,zero }}$ values are observed with $\mathrm{NH}_{2}$-terminal truncations. The deletion of the first 30 amino acids generates channels with $\mathrm{K}_{1 / 2 \mathrm{ATP}}$ of $\sim 100-120 \mu \mathrm{M}$ and a $\mathrm{P}_{\mathrm{o}, \text { zero }}$ of $\sim 0.9$ (Babenko et al., 1999; Koster et al., 1999b; Reimann et al., 1999b), and this shift in nucleotide sensitivity may be sufficient to cause dramatic changes in the physiology of cells in which it is expressed (Koster et al., 2000). These reduced ATP sensitivity, increased $\mathrm{P}_{\mathrm{o}, z e r o}$ phenotypes are very similar to that we observe for R16A mutant channels, suggesting that loss of the positive charge at residue 16 may underlie the behavior of the truncated channels.

\section{Possible Structure of the Cytoplasmic Domains}

Previous experiments have demonstrated direct interaction of isolated $\mathrm{COOH}$-terminal fragments of Kir channels with PIP $_{2}$ (Huang et al., 1998; Soom et al., 2001) and that the $\mathrm{COOH}$ terminus associates with the cell membrane in a $\mathrm{PIP}_{2}$ regulated manner (Cukras et al., 2002). The data suggest the presence of a common Kir lipid-interacting (KIRLI) domain that may be structurally related to $\mathrm{PIP}_{2}$-interacting $\mathrm{PH}$ domains (Harlan et al., 1995; Lemmon et al., 1996; Shaw, 1996; Touhara et al., 1995). We proposed that the KIRLI domain is assembled primarily from amino acids $170-320$ of the $\mathrm{COOH}$ terminus, but that the first $\beta$-strand $(\beta 1)$ may be contributed by the $\mathrm{NH}_{2}$ terminus (Cukras et al., 2002). Fig. 7 shows secondary structure predictions for the $\mathrm{NH}_{2}$ terminus from several prediction algorithms. Two $\beta$-strands (residues 34-38, 42-46) are predicted in the beginning of the conserved region of the $\mathrm{NH}_{2}$ terminus. Conceivably one of these may form $\beta 1$ of the proposed KIRLI domain (Cukras et al., 2002) and this is consistent with the association of isolated $\mathrm{NH}_{2}$-terminal fragments that include this segment with the $\mathrm{COOH}$ terminus (Jones et al., 2001). The residues we 
identify as potentially affecting ATP binding (K47, R50, R54) all cluster in a loop between the second proposed $\beta$-strand and a preM1 extended helix. While high-resolution structural information is ultimately required, we may speculate that this loop additionally combines with residues in the $\mathrm{COOH}$ terminus to form an ATP binding pocket.

This work was primarily supported by National Institutes of Health grant HL54171 (to C.G. Nichols), as well as by a National Institutes of Health Cardiovascular Training grant (T32 HL07275, fellowship support of C.A. Cukras), and the Washington University NIH DRTC (DK20579).

Submitted: 6 May 2002

Revised: 24 July 2002

Accepted: 30 July 2002

\section{R E F E R E N C E S}

Babenko, A.P., G. Gonzalez, and J. Bryan. 1999. The N-terminus of KIR6.2 limits spontaneous bursting and modulates the ATP-inhibition of KATP channels. Biochem. Biophys. Res. Commun. 255:231238.

Baukrowitz, T., U. Schulte, D. Oliver, S. Herlitze, T. Krauter, S.J. Tucker, J.P. Ruppersberg, and B. Fakler. 1998. PIP2 and PIP as determinants for ATP inhibition of KATP channels. 282:1059-60. Science. 282:1141-1144.

Boim, M.A., K. Ho, M.E. Shuck, M.J. Bienkowski, J.H. Block, J.L. Slightom, Y. Yang, B.M. Brenner, and S.C. Hebert. 1995. ROMK inwardly rectifying ATP-sensitive $\mathrm{K}+$ channel. II. Cloning and distribution of alternative forms. Am. J. Physiol. 268:F1132-F1140.

Clement, J.P.T., K. Kunjilwar, G. Gonzalez, M. Schwanstecher, U. Panten, L. Aguilar-Bryan, and J. Bryan. 1997. Association and stoichiometry of K(ATP) channel subunits. Neuron. 18:827-838.

Cukras, C.A., I. Jeliazkova, and C.G. Nichols. 2002. Structural and functional determinants of conserved lipid interaction domains of inward rectifying Kir6.2 channels. J. Gen. Physiol. 119:581-591.

Drain, P., L. Li, and J. Wang. 1998. KATP channel inhibition by ATP requires distinct functional domains of the cytoplasmic C terminus of the pore-forming subunit. Proc. Natl. Acad. Sci. USA. 95: 13953-13958.

Enkvetchakul, D., G. Loussouarn, E. Makhina, and C.G. Nichols. 2001. ATP interaction with the open state of the K(ATP) channel. Biophys. J. 80:719-728.

Enkvetchakul, D., G. Loussouarn, E. Makhina, S.L. Shyng, and C.G. Nichols. 2000. The kinetic and physical basis of K(ATP) channel gating: toward a unified molecular understanding. Biophys. J. 78: 2334-2348.

Fan, Z., and J.C. Makielski. 1997. Anionic phospholipids activate ATP-sensitive potassium channels. J. Biol. Chem. 272:5388-5395.

Fan, Z., and J.C. Makielski. 1999. Phosphoinositides decrease ATP sensitivity of the cardiac ATP-sensitive $\mathrm{K}(+)$ channel. A molecular probe for the mechanism of ATP-sensitive inhibition. J. Gen. Physiol. 114:251-269.

Harlan, J.E., H.S. Yoon, P.J. Hajduk, and S.W. Fesik. 1995. Structural characterization of the interaction between a pleckstrin homology domain and phosphatidylinositol 4,5-bisphosphate. Biochemistry. 34:9859-9864.

Hilgemann, D.W., and R. Ball. 1996. Regulation of cardiac $\mathrm{Na}+, \mathrm{Ca} 2+$ exchange and KATP potassium channels by PIP2. Science. 273:956-959.

Huang, C.L., S. Feng, and D.W. Hilgemann. 1998. Direct activation of inward rectifier potassium channels by PIP2 and its stabilization by Gbetagamma. Nature. 391:803-806.
Inagaki, N., T. Gonoi, J.P. Clement, N. Namba, J. Inazawa, G. Gonzalez, L. Aguilar-Bryan, S. Seino, and J. Bryan. 1995a. Reconstitution of IKATP: an inward rectifier subunit plus the sulfonylurea receptor. Science. 270:1166-1170.

Inagaki, N., T. Gonoi, and S. Seino. 1997. Subunit stoichiometry of the pancreatic beta-cell ATP-sensitive $\mathrm{K}+$ channel. FEBS Lett. 409:232-236.

Inagaki, N., Y. Tsuura, N. Namba, K. Masuda, T. Gonoi, M. Horie, Y. Seino, M. Mizuta, and S. Seino. 1995b. Cloning and functional characterization of a novel ATP-sensitive potassium channel ubiquitously expressed in rat tissues, including pancreatic islets, pituitary, skeletal muscle, and heart. J. Biol. Chem. 270:5691-5694.

Jones, P.A., S.J. Tucker, and F.M. Ashcroft. 2001. Multiple sites of interaction between the intracellular domains of an inwardly rectifying potassium channel, Kir6.2. FEBS Lett. 508:85-89.

Koster, J.C., B.A. Marshall, N. Ensor, J.A. Corbett, and C.G. Nichols. 2000. Targeted overactivity of beta cell K(ATP) channels induces profound neonatal diabetes. Cell. 100:645-654.

Koster, J.C., Q. Sha, and C.G. Nichols. 1999a. Sulfonylurea and $\mathrm{K}(+)$-channel opener sensitivity of K(ATP) channels. Functional coupling of Kir6.2 and SUR1 subunits. J. Gen. Physiol. 114:203213.

Koster, J.C., Q. Sha, S. Shyng, and C.G. Nichols. 1999b. ATP inhibition of KATP channels: control of nucleotide sensitivity by the N-terminal domain of the Kir6.2 subunit. J. Physiol. 515:19-30.

Lederer, W.J., and C.G. Nichols. 1989. Nucleotide modulation of the activity of rat heart $\mathrm{K}_{\mathrm{ATP}}$ channels in isolated membrane patches. J. Physiol. 419:193-211.

Lemmon, M.A., K.M. Ferguson, and J. Schlessinger. 1996. PH domains: diverse sequences with a common fold recruit signaling molecules to the cell surface. Cell. 85:621-624.

Li, L., J. Wang, and P. Drain. 2000. The I182 region of K(ir)6.2 is closely associated with ligand binding in K(ATP) channel inhibition by ATP. Biophys. J. 79:841-852.

Liou, H.H., S.S. Zhou, and C.L. Huang. 1999. Regulation of ROMK1 channel by protein kinase A via a phosphatidylinositol 4,5-bisphosphate-dependent mechanism. Proc. Natl. Acad. Sci. USA. 96:5820-5825.

Neher, E., and C.F. Stevens. 1977. Conductance fluctuations and ionic pores in membranes. Annu. Rev. Biophys. Bioeng. 6:345-381.

Proks, P., F.M. Gribble, R. Adhikari, S.J. Tucker, and F.M. Ashcroft. 1999. Involvement of the N-terminus of Kir6.2 in the inhibition of the KATP channel by ATP. J. Physiol. 514:19-25.

Reimann, F., T.J. Ryder, S.J. Tucker, and F.M. Ashcroft. 1999a. The role of lysine 185 in the Kir6.2 subunit of the ATP-sensitive channel in channel inhibition by ATP. J. Physiol. 3:661-669.

Reimann, F., S.J. Tucker, P. Proks, and F.M. Ashcroft. 1999b. Involvement of the n-terminus of Kir6.2 in coupling to the sulphonylurea receptor. J. Physiol. 518:325-336.

Rohacs, T., J. Chen, G.D. Prestwich, and D.E. Logothetis. 1999. Distinct specificities of inwardly rectifying $\mathrm{K}(+)$ channels for phosphoinositides. J. Biol. Chem. 274:36065-36072.

Schulte, U., H. Hahn, H. Wiesinger, J.P. Ruppersberg, and B. Fakler. 1998. pH-dependent gating of Romk (Kir1.1) channels involves conformational changes in both $\mathrm{N}$ and $\mathrm{C}$ termini. J. Biol. Chem. 273:34575-34579.

Shaw, G. 1996. The pleckstrin homology domain: an intriguing multifunctional protein module. Bioessays. 18:35-46.

Shyng, S., T. Ferrigni, and C.G. Nichols. 1997a. Control of rectification and gating of cloned KATP channels by the Kir6.2 subunit. J. Gen. Physiol. 110:141-153.

Shyng, S., T. Ferrigni, and C.G. Nichols. 1997b. Regulation of KATP channel activity by diazoxide and MgADP. Distinct functions of the two nucleotide binding folds of the sulfonylurea receptor. $J$. Gen. Physiol. 110:643-654. 
Shyng, S., and C.G. Nichols. 1997. Octameric stoichiometry of the KATP channel complex. J. Gen. Physiol. 110:655-664.

Shyng, S.L., C.A. Cukras, J. Harwood, and C.G. Nichols. 2000. Structural determinants of $\operatorname{PIP}(2)$ regulation of inward rectifier K(ATP) channels. J. Gen. Physiol. 116:599-608.

Shyng, S.L., and C.G. Nichols. 1998. Membrane phospholipid control of nucleotide sensitivity of KATP channels [see comments]. Comment in: Science 1998 Nov 6;282(5391):1059-60. Science. 282:1138-1141.

Sigworth, F.J. 1980. The variance of sodium current fluctuations at the node of Ranvier. J. Physiol. 307:97-129.

Soom, M., R. Schonherr, Y. Kubo, C. Kirsch, R. Klinger, and S.H. Heinemann. 2001. Multiple PIP2 binding sites in Kir2.1 inwardly rectifying potassium channels. FEBS Lett. 490:49-53.

Tanabe, K., S.J. Tucker, M. Matsuo, P. Proks, F.M. Ashcroft, S. Seino, T. Amachi, and K. Ueda. 1999. Direct photoaffinity labeling of the Kir6.2 subunit of the ATP-sensitive $\mathrm{K}+$ channel by 8 -azidoATP. J. Biol. Chem. 274:3931-3933.

Touhara, K., W.J. Koch, B.E. Hawes, and R.J. Lefkowitz. 1995. Mutational analysis of the pleckstrin homology domain of the betaadrenergic receptor kinase. Differential effects on $\mathrm{G}$ beta gamma and phosphatidylinositol 4,5-bisphosphate binding. J. Biol. Chem. 270:17000-17005.
Tucker, S.J., and F.M. Ashcroft. 1999. Mapping of the physical interaction between the intracellular domains of an inwardly rectifying potassium channel, Kir6.2. J. Biol. Chem. 274:33393-33397.

Tucker, S.J., F.M. Gribble, P. Proks, S. Trapp, T.J. Ryder, T. Haug, F. Reimann, and F.M. Ashcroft. 1998. Molecular determinants of KATP channel inhibition by ATP. EMBO J. 17:3290-3296.

Tucker, S.J., F.M. Gribble, C. Zhao, S. Trapp, and F.M. Ashcroft. 1997. Truncation of Kir6.2 produces ATP-sensitive K+ channels in the absence of the sulphonylurea receptor. Nature. 387:179183.

Varnum, M.D., and W.N. Zagotta. 1997. Interdomain interactions underlying activation of cyclic nucleotide-gated channels. Science. 278:110-113.

Woodward, R., E.B. Stevens, and R.D. Murrell-Lagnado. 1997. Molecular determinants for assembly of G-protein-activated inwardly rectifying K+ channels. J. Biol. Chem. 272:10823-10830.

Zagotta, W.N., and S.A. Siegelbaum. 1996. Structure and function of cyclic nucleotide-gated channels. Annu. Rev. Neurosci. 19:235263.

Zhou, H., S.S. Tate, and L.G. Palmer. 1994. Primary structure and functional properties of an epithelial K channel. Am. J. Physiol. 266:C809-C824. 